\title{
Index
}

Abandonment, as national phobia, 79-82, $262 n 7,292 n 7$

Agricultural rites, 102, 131-133, 278n12

Agricultural year, 100-103

Agriculture, Ministry of, 15, 27, 78, 151, 268n9

Alcohol:

consumption of, 103, 105, 203, 268n10 manufacture of, 146

Amulets, 107, 117, 270n16, 271n18, 274n26. See also Good luck charms; Medicine, traditional; Occult powers.

Animal Health and Husbandry, Department of, 179

Authority:

Buddhist, 121-122

household, 48-50, 273n 23

Moslem, 70, 261n10

of patrons, $62,118,258 \mathrm{n} 1,282 \mathrm{n} 22$

See also Government; Kingship.

Ayal, Eliezer, 15

Ayutthayā, 38, 80, 93, 184, 232

Bam Phen, 100, 104, 152, 267n8

Bam Phen Temple, 66, 67, 94, 259n4

Bang Chan:

administrative divisions of , 23, 110-112

appearance of, at various periods, 20-22, $36,89-90,98,122,142$

defined for study, 22-23

identity of, 135, 140-142, 280n16

Bang Chan Study. See Cornell University Thailand Project.

Bāngkapi, 23, 43, 127, 140, 198

Bāngkhen, 172

Bangkok, 19, 42, 64, 266n4

growth of, 74-76

intercourse with, 124, 190-193, 199-202, 205-207, 225-226, 274n 1, 275n4 markets in, $65,176-177$

relations with, 124-125, 199-202, 205-207, 225-227

Bāngkrabȳ, 89, 192

Bāng Pa Kong River, 39, 233, 253n7, 254n8

Bang Sȳ, 114

Banks, 188

Bastian, Adolf, 32, 83, 259n6

Bentham, Jeremy, 83, 263n10

Bock, Carl, 40

Bōdin, Chao Phrayā, 254n8

Boon-Long, Siribongse, 16, 83

Bowring, Sir John, 36

Bradley, Dan Beach, 64, 75

Bride price, 47, 152

Brook, Sir James, 83

Buddha, teachings of, 92-93, 95, 97, 122, $184,265 \mathrm{n} 1$. See also Buddhism.

Buddha's Birthday, 36, 103, 290n5

Buddhism:

and concept of death, 290n 5

and idea of history, 92-93, 212, 265n2

teachings of, 95, 144, 292n6. See also

Buddha, teachings of.

Buffalo:

care of, 116, 117, 133, 179

in economic exchange, $68,69,137,147$, 169

and government programs, 169, 179

as meat, $273 \mathrm{n} 22,286 \mathrm{n} 8$

reach Bang Chan, 68, 99, 213

Bunnag, Chuang. See Srisurijawong, Chao Phrayā.

Bunnag, Dit. See Phraklāng, Chao Phrayā; Srisurijawong, Somdet Chao Phrayā.

Bunnag, Nàj Chyd, 40

Bunnag, That. See Srīphipat Rātanarāchakosa, Phrayā. 
Burial of dead, 63, 90. See also Cremation. Burma, 38, 93, 181, 217

\section{Calendar, 268n11}

Cambodia, 38, 180, 216, 254n 8

Canals, 39, 43, 75, 126, 254n8, 256n 11

Cash economy, conversion to, 134 , 160-161, 289n19. See also Markets.

Central plains, 35-36

Chachoengsao, 140

Chandruang, Kamut, 275n4

Chiangmai, 78

Childbirth, 48, 54, 160

China, 184, 207

Chinese:

occupations of, $39,42,76,87,93,134$, $137,156,177,263 \mathrm{n} 12,264 \mathrm{n} 14$

propaganda against, 156, 184

settlements of, 42,93

wages of, 264n 13

Chulalongkorn the Great. See Rama V.

Chyn the martinet, 107-110, 112, 118, $219,220,221$

Class stratification, 205

Commerce, Ministry of, 149-150

Commercialization. See Markets.

Commune (tambon), 23, 110-111

Commune headman (kamnan), 23, 76, 77, 198

duties of, $77,112,114,127,281 \mathrm{n} 21$

selection of, 112-114

succession of, $198,287 \mathrm{n} 12$

See also Phlym, Kamnan.

Communications, 172, 174, 180, 181, 204. See also Movies; Radio.

Communism, 184, 189

Comparative studies of changing culture, 26

Concepts, translation of, 32-34

Contracts, 258n 1

"Cooked and raw," 145, 159-60

Cornell University Thailand Project: effects of, on Bang Chan, 179-181, 188-190, 198

personnel of, 13-14

research objectives of, 26-30

Corvée, 64, 99-100, 130

tax in lieu of, 129

Costume, 19-20, 214

Coup d'état (1949), 171-172

Courtship, 104

Credit:

on future services, 86-87. See also Slaves, debt.

offered by traders, 177

See also Loans.

Cremation, 86, 164-166, 199, 289n $1 \mathrm{n} 2$

costs of, 165, 290n3n6 season for, 103, 119

See also Burial of dead.

Crop failure, 71, 103, 268n9

Damrong, Prince (Damrong Rāchānuphāb, Somdet Krom Phrayā), 91, 110, 128, 265n2, 275n5

Death, concept of, 290n5

De Young, John E., 15, 27, 288n 17

Dilock, Prince, 83, 257n19, 259n3, $263 \mathrm{n} 10,264 \mathrm{n} 13$

District (amphoe) $, 23,31,88,272 \mathrm{n} 21$

District education of ficer, 188

District officer (nāj amphoe), 112, 116, 125, $150,169,198,278 \mathrm{n} 8$

Don Myang airport, 20

Drought, 134

Ducks and chickens, 155

Earth Guardian (Phra Phūm), 133

Education. See Schools.

Education, Ministry of, 15, 189, 190

Elections, 150, 171, 203

Elephants, 45, 61, 63

Elman, Emily, 16

Emarun, Aram, 14, 15

Family, 52, 257n19

status within, 49, 56, 273n23

See also Households; Kinship.

Family name edict, 130, 278n11

Farm income, 285n 5

Farming, 100-103, 136-137. See also Irrigation.

Fertilizer, 180, 187-188, 195

Festivals, 164-168, 289n1n2, 290n3n4

cost of, $281 \mathrm{n} 18 \mathrm{n} 19,290 \mathrm{n} 4$

See also Buddha's Birthday; Cremation; Laūj Krathong; Lent, Buddhist; Marriage; New Year festival; Priests, ordination of; Teachers, homage to; Tonsure ceremony.

Fine Arts Department, 100, 108

Firemaking, 214

Fireworks Department, 100, 108

Fish, 60, 65, 253n4 market prices of, $65,259 \mathrm{n} 3$

Fishing, 45, 93, 94, 123, 174

abandonment of, 134

devices for, 21, 44, 65

Fish ponds, 174-175, 179, 205

Floods, 117, 133, 157, 268n9, 288n16

Flora, 24, 253n3n 5

Folk-urban thesis, 30-31

France, 181

Free enterprise, 82-85, 87-89, 123-124, $161,263 \mathrm{n} 10$ 
Gambling, 85, 86, 92, 101, 105, 138-139, 194

edict against, 127-128, 130

Gambling games, 269n12

Gasoline engines, 154, 174, 176, 195, 215, $219,288 \mathrm{n} 15$

Gerini, G. E., 288n 18

Goldsen, Rose K., 14, 180, 187, 195

Good luck charms, 269n12

Government:

and control of population, 64, 80-85, $156,230-232,261 \mathrm{n} 10,262 \mathrm{n} 6,264 \mathrm{n} 16$

effective jurisdiction of, 62, 64-65, $275 \mathrm{n} 5$

organization of, $23,62,110,272 \mathrm{n} 21$

reorganization of, 84-85, 91, 125-126, $282 \mathrm{n} 22$

See also Kingship.

Government programs, 133, 150-152, 156 concerning land, 77-79, 262n5

Graham, W. A., 127

Guardian spirit (Phra Phūm), 78

Haas, Mary R., 14

Hallowell, A. Irving, 292n4

Hamlets (mūbann), 23, 60-73, 89-90, 98-110, 266n6

cooperation within, 61-63, 98, 100-103, 136-139

dispersal of, 71-73, 120, 204-205

growth of, 104-105, 114-115, 274n24

intermarriage within, 104, 270n14

membership in, 56, 60-61, 260n9, 266n7, $274 n 24$

organization of, $261 \mathrm{n} 10$

sanctions in, 105-108

See also Laotian settlements; Moslem hamlet; Prisoners of war, hamlets of.

Hamlet headman ( $p b \bar{u}$ jaj bān), 23, $110-112,188,198$

duties of, 31, 169-170, 179

Hanks, Jane R., 14, 15, 28

Hanks, Lucien M., 15, 28, 29, 262n8

Harvest, 136-137

Harvest festival, 101-102, 164

Hauck, Hazel M., 14, 15, 180, 256n 15

Hendry, James B., 15

Herodotus, 31, 33

History, concept of, 31-34, 291nn1-2 defined, 208

as ebb and flow of prosperity, 213-217

a s effects of unique events, 232-235

as explanation of problematic events, 208-211

as force and process, 222-227

as organic metamorphosis, 227-235, 294nn8-10

as work of heroes, 217-222
Hongladaromp, Tasanee, 16

Household compound, 115-116

Household production, 136-138, 146, 195-196

Households:

cash income of, $281 \mathrm{n} 19,285 \mathrm{n} 5,287 \mathrm{n} 9$

cooperation between, 55-57, 60-66, 98, 105-106, 273n23, 280n17, 285n6

membership of, $52,66,258 \mathrm{n} 24,262 \mathrm{n} 7$

migrant, 104-105

polygynous, 115-116

work requirements of, 44-45, 148-149, $256 \mathrm{n} 15$

House styles, 43, 60, 137, 256n13

Hualamphong, 89

Hunting, 57, 258n 25

Immigration, 262n9

Ingersoll, Jasper C., 15

Ingram, James C., 15, 263n12

Inheritance, 48-49, 178-179, 256n17, $287 \mathrm{n} 13$

Interior, Ministry of, 15, 91

Irrigation, $84,103,117,126,187-188$

devices for, 148, 155-156, 213, 215, 220, $288 \mathrm{n} 15$

Janlekha, Kamol, 14, 15, 24, 25, 27, 28, $179,227,256 \mathrm{n} 14,257 \mathrm{n} 20,290 \mathrm{n} 4$, $290 \mathrm{n} 7$

Japanese occupation, 157-158, 168-169, 184

Kamnan. See Commune headman; Phlym, Kamnan.

Kant, Immanuel, 210

Kaufman, Howard, 15

Keyes, Charles F., 15

Khā, 35, 57-58, 110, 253n6

Kingshill, Kon rad, 15

Kingship:

and absolute power, $150,275 \mathrm{n} 5,282 \mathrm{n} 22$

and indirect rule, 125

and land possession, 78-79

Kinship, 45-56

and ancestors, 258n23, 279n13

and household cooperation, 273n23, 280n17, 285n6

and inheritance, $155,287 \mathrm{n} 13$

a s model for interpersonal relations, $257 \mathrm{n} 21$

and participation in rites, 283n1, 290n4

Kinship obligations, termination of, $278 \mathrm{n} 8$, $284 \mathrm{n} 2$

Kinship terms, 256n16, 257n18, 258n23, 260n $8,275 \mathrm{n} 2$

change in usage of, $223,291 \mathrm{n} 9$

Kirsch, Thomas, 284n 3 
Kongsa, Jadun, 14

Korea, 180

Krainin, Ewing, 16

Labor:

child, $65,137,148-149,155$

exchange of, 63, 98, 116, 136-137, 194, $196,224,280 \mathrm{n} 17$

hiring of, $63,135,169,179,189,194$

kinship in acquisition of, 45-47, 137, $280 \mathrm{n} 17$

for rice cultivation, $43-45,148-149$, $265 \mathrm{n} 14$

scarcity of, $45,148,157$

sources of, 25-26, 118, 135, 147, 178, 263n12, 278n12

See also Rentals, land; Wages.

Lajonquière, E. Lunet de, 87

Land:

area cultivated, $67,86-87,105,146,179$

sales of to Bangkok, 194, 199-200, 202

as security for debt, 90, 146-147, 270n 15

vacant, $35,87,89,222-223$

Land claims, 39, 57, 76-79, 85, 90-91, $265 \mathrm{n} 17$

Land disputes, 77, 90-91, 153-154, $265 \mathrm{n} 17$

Landholding, 78-79, 262nn4-5

absentee, 39-40, 89, 199-200

in Bangkok, 75-76, 261n1

and certification of ownership, 76-79, 91,129

percentage of, 24-25

and size of holdings, 39, 76-77, 85, 89, $113,117,120,123,124,178,261 \mathrm{n} 2$, $278 \mathrm{n} 12$

Land prices, 77, 97, 114, 117, 147, 260n7 by decade, 243

Lands, Department of, 77, 78

Laos, 38, 180

Laotian carpenters, 87, 264n 15

Laotian settlements:

in Bang Chan, 66-70, 136, 203, 204, 220

dispersal of, 73

identity of, 136, 280n16

on Saen Saeb canal, 40-42

Lauj Krathong, 36

Lent, Buddhist (Phansā), 101, 103, 144

Lerner, Daniel, 293n17

Loans:

interest on, 177-178, 270n 15

secured by land, $86,147,153,285 \mathrm{n} 5$

settlement of, $85,86,153,154$

sources of, 146, 153, 177, 194, 201, $285 \mathrm{n} 6$

Lōlae, 177, 256n10, 267n8

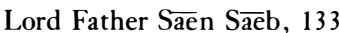

Lord of the Land, 131, 132, 142

Lord of the Place (Chao Thì), 63, 131, $132,142,145,160$

McWaters, Jean, 16

Malay rajahs, 38, 229

Mari, 122-123

Markets, 58, 59, 65, 134, 267n8

dependence on, 57-59, 65

organization of, 123-124, 274n 1

selling in, 225, 274n 1

wartime, 158, 169, 224

See also Stores; Traders.

Marriage, 50-52

annulled by parent, 107

and bride price, 47,152

Buddhist-Moslem, 197

between hamlets, 104-105, 270n14

plural, 115-116

and separation, 115

Medical Department, 100

Medicine:

and concept of human body, 284n 3

modern, 179-180

traditional, 93, 98, 265n $3,279 \mathrm{n} 13$

Merit:

attainment of, 59, 60, 92-97, 121-122, $124,272 \mathrm{n} 20,273 \mathrm{n} 3$

and expiation of $\sin , 159-160$

and karma, 59, 155

Merit making: by building temples, 97-98, 103, 140, $173,197-198,291 \mathrm{n} 10$

by feeding priests, 22, 109, 118

by giving money and services, 140, 197

by ordaining son, $47,283 \mathrm{n} 1$

by presenting robes to priests, 197

by religious devotion, 129, 159, 266n 4

Metah, Singto, 14, 15, 27, 28, 179

Middlemen, 195

Migration, 24, 74, 85-86, 88, 104-105, $263 \mathrm{n} 12,290 \mathrm{n} 8$

to Bangkok, 124, 173, 192-193, 199-202, 225

into country, 74, 85-86, 90-91, 158-159, 197-198, 202

among renters and farm workers, $104-105,178,290 \mathrm{n} 7$

Military service, 124, 130, 157, 192, 200, $278 \mathrm{n} 10$

Minburī, 19, 31, 135, 197

as province and district, $23,110,140$, 205, 198, 272n21, 287n10

market in, 123, 177, 267n 8

rice mills in, 135

schools in, 193, 199 
Misfits, 105-108, 271n18, 292n6

Modernization, 293n7

Money:

earning of, 146-147, 157-159, 174-178, 194-196, 287n9

management of, 134, 153, 160-161, $289 \mathrm{n} 19$

sources of, 86, 137, 146, 177, 194, 224

Mongkut, king. See Rama IV.

Moore, Frank J., 15

Moslem hamlet, 23, 58, 59, 122-123, 219

dispersal of, 70-73, 112, 203

organization of, $26 \ln 10$

settlement of, 39-40, 77

Moslems, 29, 184

and intermarriage with Buddhists, 196-197, 273n22

male, in Bang Chan, by age (table), 242

and relations with Buddhists, 118 , 273n22, 286n8

Mother Earth (Māe Thōrani), 78, 173, 186

Mother Splendor $(\mathrm{M} \overline{\mathrm{ae}} \mathrm{Si}), 103,116$

Movies, 179, 184, 192

Murder, 115, 270n16

Murdock, George P., 257n21

Nāj Loet Setthabutr, 126, 130, 277n6

Nakāun Nāyok Province, 104, 140, 184

Nakteng (rogues), 106, 139, 27 $\ln 17$

Nakorn, Yaūd (Khru Jaüd), 14, 28

Nationalism, 156, 172, 184, 190-192

Neighborhood, 194, 223, 224. See also Hamlet.

New Year festival (Song Krān), 36, 103, $116,269 \mathrm{n} 13$

Notton, Camille, 265n2

Novices, Buddhist (nēn), 266n5. See also Priests.

Occult powers, $117,118,27 \ln 18,274 \mathrm{n} 26$

Occupations, 24-26, 30-31, 161-162, 174-176, 195-196, 263n12

in Bangkok, 87, 264n 15

new, 176, 192-193, 225

Orchards, 113, 119

Ordination. See Priests.

Pallegoix, Msgr. Jean Baptiste, 36

Patron-client relationships, 50, 62, 82-83, 118, 199-200, 204, 282n22

Buddhist view of, 121-122

at hamlet level, 141-142

in markets, 123-124

and social order, $262 \mathrm{n} 8,263 \mathrm{n} 12$

Pattani, 122

Peddlers. See Traders.

Phillips, Herbert P., 14, 15, 145, 257n19, 274n1, 284n4, 289n19

Phlym, Kamnan, 97, 110-120, 122, 129, $135,140,141,146,150,152,189$, $192,193,196,198,199,203,204$, $217,219,221,281 \mathrm{n} 18$

Phra Khanong, 43

Phraklāng, Chao Phrayā (Dit Bunnag), 39, $75,254 \mathrm{n} 8,255 \mathrm{n} 9$. See also

Srisurijawong, Somdet Chao Phrayā.

Phranaukāun (Bangkok Province), 150, $287 \mathrm{n} 10$

Phrapadaeng, 99, 287n13

Phrayā Korani, 122

Police, 127-128, 172

Department of, 127-128, 172

Polygyny, 115-116

Population:

of Bang Chan, 23-25

registration of, 80-82

of Thailand (1850), 253n7

Population pressure, 76-77, 85, 222-225

Priests, 21-22, 36

head, 140-141, 279n13, 291n10

males ordained as, by age group (table), 242

and monastic discipline, 144-145, 159-160, 294n 3

ordination of, 47, 66, 103, 143-144, 199, $28 \ln 19,284 n 3$

role of, in community, 95-97, 140-141, $265 \mathrm{n} 3$

as teachers, 29, 128-129

Prisoners of war, hamlets of, 66-68, $71-73,255 \mathrm{n} 9$

Progress:

in merit, 216-217, 292n6

in "new culture," 190-192

in technology, 214-217

and Western influence, 226-227

Provincial governor ( $p h \bar{u} w \bar{a}$ răchakān myang), 110

Public Health, Ministry of, 15, 180

Rabibhadana, Akin, 15

Radcliffe-Brown, A. R., 294n8

Radio, 29, 156, 172, 174, 180-181, 184, 219,221

Railway Department, 192

Rains, 100-101, 268n9

Rajadhon, Phrayā Anuman, 16

Rajatasilpin, Anusith, 15

Ralis, Max, 14, 180, 187, 195

Rāma Kamgheng, king, 162

Rama I, king, 38, 74, 229

Rama II, king, 80, 82, 264n 16

Rama III, king, 38, 39, 83, 122, 254n8, $255 \mathrm{n} 9$ 
Rama IV, king (Mongkut), 39, 64, 82, 83-84, 87, 263n12, 265n16

Rama V, king (Chulalongkorn the Great), 66-67, 84-85, 99, 123, 258n1, 264n 16 and education, 128

and government reorganization, 84, 125 names Bang Chan temple, 109

Rama VI, king (Vajiravudh), 99, 126, $275 n 3,28 \ln 21$

gambling edict of, 127, 130

Rama VII, king (Prajadhipok), 149

Rangsit canal, 84, 103

Ratchaburī (Radburi), 40, 70

Redfield, Robert, 30, 294n9

Religious af filiation (table), 242

Rentals, equipment, 176

Rentals, land, 75, 105, 135, 153, 178, 260n7

income from, 153, 260n7

and labor supply, 179

payment of, in grain, 153,155, 178

prices of, by decade, 243

and tenant income, 195

Revolution of 1932, 150

Rice:

area cultivated in, $67,69,105,135$

cost of producing, 194-195, 285n 5

experiments with, 186-187

governmental assistance for, 150 , 151-152, 186-188

hazards for crop, 45, 65, 71, 90, 101, $103,133-134,213,268 \mathrm{n} 9$

as medium of exchange, 138-139

prices of, 82, 134, 147, 153, 168, 169, $280 \mathrm{n} 15,28 \ln 19$

prices of, by decade, 243

rites for, 102, 131-133, 278n 12

as symbol, $162,288 \mathrm{n} 18$

techniques for growing, 44, 65, 68-69, $99,147-149,180,187-188,213-215$

yields of, 147-148, 187-188, 281n19, $285 \mathrm{n} 7$

Rice Mills, 135, 184

Rice Mother (Māē Pōsop), 55, 78, 101, $132,142,149,160,278 \mathrm{n} 12$

Rituals. See Agricultural rites; Buddha's

Birthday; Cremation; Festivals; Laūj

Krathong; Lent, Buddhist; Marriage;

New Year festival; Priests, ordination of; Tonsure ceremony.

Roads, 168-169, 174, 176, 189, 234-235

Robbery, 117, 127, 173

Sād festival, 164

Saengmani, Vichitr, 14, 16, 259n 5

Saen Saeb canal:

construction of, $39,254 \mathrm{n} 8$ dredging of, 126

Saen Saeb plain, 35-36

early settlements on, 39-42, 233

Sakdi (sakdinā $), 58,26 \ln 3$

Sāmsen, 42, 89, 114, 192, 202

San Pong, 27

Sarabaja, Loebongse, 14, 15, 259n4, $272 \mathrm{n} 21$

Schools, 23

attitudes toward, 129, 151, 187, 192-193, 225-226, 287n14

attendance at, $151,239,277 \mathrm{n} 7$

in Bangkok, 99, 128, 193, 226

Buddhist, 91, 128-129, 150, 272n21, $277 \mathrm{n} 7$

government-sponsored, 150, 188-190, $287 \mathrm{n} 11$

See also Students; Teachers.

Self-continuity, 292n 4

Settlement of population:

in Bang Chan, 35, 43, 66, 256n12

in Bangkok, 74-76

between hamlets, 135, 136-137, 266n6

in hamlets, 85-86, 98-99, 104-105, 112, 114,135

in Sae $\mathrm{n}$ Sae b region, 39-42, 233, 256n10

Sharp, Lauriston, 15, 28, 29, 179, 180, 227, 258nn23-24

Siam Canal and Irrigation Company, 84

Siam Society, 15

journal of, 28

Sin, expiation of, $145,159-60$

Sin, Uncle, 93-98, 109, 112, 122, 124, $152,155,219,220$

Skinner, G. William, 15, 42, 263n12

Slaves, 58

as carpenters in Bangkok, 264n15

debt, 86-87, 107, 116, 274n 25

liberation of, 66-67, 70, 81, 84, 86, 88, $123,230,260 \mathrm{n} 7$

Smith, Adam, 46, 57, 161

Social change, judgment of, 210-211

Social history, concept of, 30-34

Social mobility, 104, 105, 122-124, 126, 130, 154-155, 288n 14, 293n7

through affiliation, 124-125, 199-204

through education, 192-193

Songkrām, Field Marshal Pibul, 184

Spirit patrons, 131-133, 160, 278n12, $280 \mathrm{n} 14$

ancestral, 279n13

New Year, 269n 13

Srīphiphat Rātanarātchakosa, Phrayā (That Bunnag), 39, 254n8, 255n9

Srīsurijawong, Chao Phrayā (Chuang Bunnag), 39-40, 43, 58, 65, 66, 70, 75, 77, $83,255 \mathrm{n} 9$ 
Srīsurijawong, Somdet Chao Phrayā (Dit Bunnag), 71, 204, 255n9. See also Phraklāng, Chao Phrayā.

State. See Government.

Status:

within family, 49, 56, 273n23

occupational, 46, 161, 195-196, 293n7

sakdi, 58

urban-rural, 155, 190-193, 199-200

by wealth, $114,117,122-123,146-147$

Status symbols, 215, 226

Steward, Julian, 294n9

Stores:

in Bamphen, 100, 267n8

in Bang Chan, 20-21, 172, 174, 176-177

in Bangkok, 123

See also Markets.

Students:

desired occupation of (table), 241

scholastic attainment of (table), 240

Subankha, Heng R., 14

Subsistence economy:

changes to cash economy, 134, 160-161, $289 \mathrm{n} 19$

labor requirements of, 43-45

Sudsaneh, Saovanee, 14, 15

Suicide, 270n16

Suvanajata, Titya, 14

Tāksin, Phrayā, 38

Talismans. See Amulets; Good luck charms; Medicine, traditional; Occult powers.

Tambon. See Commune.

Taxes:

on fruit trees and gardens, 150

on land, $77,83,114,129,153,170$, $278 \mathrm{n} 9,290 \mathrm{n} 6$

in lieu of corvée, 129

poll, 64, 68, 129-130

slave's vs. freeman's, $67-68$

See also Corvée.

Teachers, 117, 118, 151, 154-155, 181, $190,199,200$

homage to (waj kbru), 118

priests as, 29, 128-129

as source of power, 98, 117, 265n 3

Temple, 21-22, 124

building of, 97-98, 108-109, 173, 207, 197-198, 272n19, 291n10

governance of, 108, 173, 188

social effects of, 94-97, 103, 107-108, 121-122, 140-141, 292n6

Temple boys (dek wat), 48, 67, 197

Temple schools, 29, 128-129, 277n 7

Temporal ordering of events, 210-213 and personal continuity, 292n4
Tenants, 179

conditions of, 105, 110-115, 134-135, 178,195

percentage of, 24-25

Textor, Robert B., 15, 258n22, 274n26, 280n 14

Thai Islam. See Moslems.

Thailand:

among neighboring states (ca. 1825), 38-39

in organic metaphor, 228-231, 294n9

population of (1850), 253n 7

self-image of, 184, 190

Thai language, transcription of, 14

Thai personality, 217-219, 266n4, 284n4, 292n6, 293n7. See also Abandonment as national phobia.

Thanyaburi, 272n21

Theatrical troupes, 118, 137-140, 165, $166,173,282 \mathrm{n} 18,283 \mathrm{n} 1$

Thewetwonwiwan, Chao Phrayā, 91

Thonburi, 108, 232, 265n3

Tonsure ceremony, 47-48, 95, 118, 137, 201, $28 \ln 18$

Traders, 19, 93-94, 123, 126, 134, 158, 174,177

and relations with suppliers and customers, 123-124, 174-177, 193, 274n1

temporary, 146

See also Markets.

Transportation:

by boat, $21,62,123,259 \mathrm{n} 2$

by bus, 19-20, 173, 174

by motorboat, $29,126,174,195-196$, $277 \mathrm{n} 6$

See also Canals; Roads.

Undertakers, $165,166,289 \mathrm{n} 2$

United States, 184, 186, 187, 259n6

University of Agricultural Science, 15 rice experimentation by, 186-187

Urbanization, 30, 225-227

Vajiravudh, king. See Rama VI

Van der Heide, J. Homan, 260n7

Vietnamese, 38

Visu Kamma, 122

Wachirayan, Prince, 128

Wages, 64, 87, 169, 264n 13

of teachers, 154

Wales, H. G. Quaritch, 275n5

Water gates (pratūnām), 19, 103, 126, 146

Wesantara, king, 143

Western influences, 226-227

on abolition of slavery, 66

on culture, 190-192 


\section{INDEX}

Western influences( cont. )

on education, 128-129, 150-151

on free enterprise, $82-85$

on government reorganization, 91 , 125-127

Wilson, David A., 15

Wittfogel, Karl, 275n5

Wonghanchao, Warin, 16

Work, annual round of, by season, 43-45, 100-103

World view, 235-236

Copernican, 181

on death and rebirth, 167

on fortune/misfortune, 92-93

on freedom/responsibility, 122-123

on suffering, 144

World War I, 224 agricultural response to, 133-134

World War II, 157, 192

recovery from, 168-169

shortages during, 158, 214

Wyatt, David K., 15, 263n10, 272n21

Xieng Khouang, 38

Youth:

and courtship, 104

and gangs, 107-108, 139-140

and leaving home, 47-48, 137-140

and recreation, 102-104, 138-139

relation of, to elders, $49,273 n 23$

Zimmerman, Carle, 285n5 